Sir,

\section{Strabismus and optic neuropathy: a complication of} retrobulbar injection

While extraocular muscle and optic nerve injury are known to occur after retrobulbar injection, simultaneous damage to both has not been reported. We report horizontal and vertical strabismus with a concurrent optic neuropathy as a complication of retrobulbar injection. The strabismus was corrected by lateral and inferior rectus recession

A broad injury pattern with a mixed clinical picture may rarely complicate retrobulbar anaesthesia.

\section{Case report}

A 77-year-old woman presented for right cataract extraction. Her pre-operative visual acuity was $6 / 18$ in each eye. The pupillary reflexes and sizes were normal and equal in both eyes. She was orthotropic with full ocular motility. She had no significant past medical history.

Routine phacoemulsification with lens implantation was carried out under local anaesthesia accomplished by a retrobulbar injection of $2 \%$ lidocaine with 1:200 000 adrenaline and $0.75 \%$ bupivacaine (1:1 ratio) with a retrobulbar needle. The volume per injection and number of local injections that had been administered could not be ascertained.

Two days following surgery the patient reported diplopia. Her best corrected vision was $6 / 24$ in the right eye and $6 / 18$ in the left eye. Three millimetres of ptosis and a $2+$ afferent pupillary defect in the right eye was noted (Fig. 1). The prism cover test in primary position revealed esotropia of 15 prism dioptres (PD) and a left hypertropia of $12 \mathrm{PD}$. The left hypertropia was greatest in upgaze. Versions and ductions demonstrated absent abduction and slight limitation of elevation in the right eye; forced ductions were normal. Fundoscopy was unremarkable in both eyes. Visual field testing revealed a superior altitudinal field defect in the right eye. Axial and coronal computed tomography of the orbit was normal.

Five months after cataract surgery, her best corrected vision was $6 / 6$ in the right eye and $6 / 18$ in the left eye. The ptosis and afferent pupillary defect persisted in the right eye. Examination at this stage revealed an exotropia of 20 PD with left hypertropia of 16 PD. Abduction in the right eye was now normal. The inferior half of the right optic nerve was pale, with the retina and its vasculature being normal (Fig. 2). The superior altitudinal scotoma remained unchanged.

Strabismus surgery was performed 7 months following phacoemulsification. Per-operatively forced duction revealed a tight inferior rectus and lateral rectus but the muscles anatomically appeared normal. An $8 \mathrm{~mm}$ lateral rectus recession and $5 \mathrm{~mm}$ inferior rectus recession of the right eye with adjustable suture technique was performed. At 4 weeks post-operatively the patient was fusing and diplopia-free in all gaze positions.

\section{Comment}

While instances of injury to the extraocular muscles ${ }^{1}$ and optic nerve ${ }^{2}$ following retrobulbar anaesthesia have been well documented, a broad injury pattern such as occurred in our patient has not been reported. The pupillary and visual field defects with the optic disc appearance in the right eye suggest an optic neuropathy, while ptosis and the pattern of strabismus implicate the levator, superior rectus and the lateral rectus muscles or their nerve supplies. The mechanism and location of injury invite speculation. Our patient's presenting features could be explained by injury to structures at the orbital apex where the optic nerve, sixth nerve and superior division of the third nerve lie in close proximity. It is also possible that the muscles and nerve or their vascular supply may have been directly traumatised by

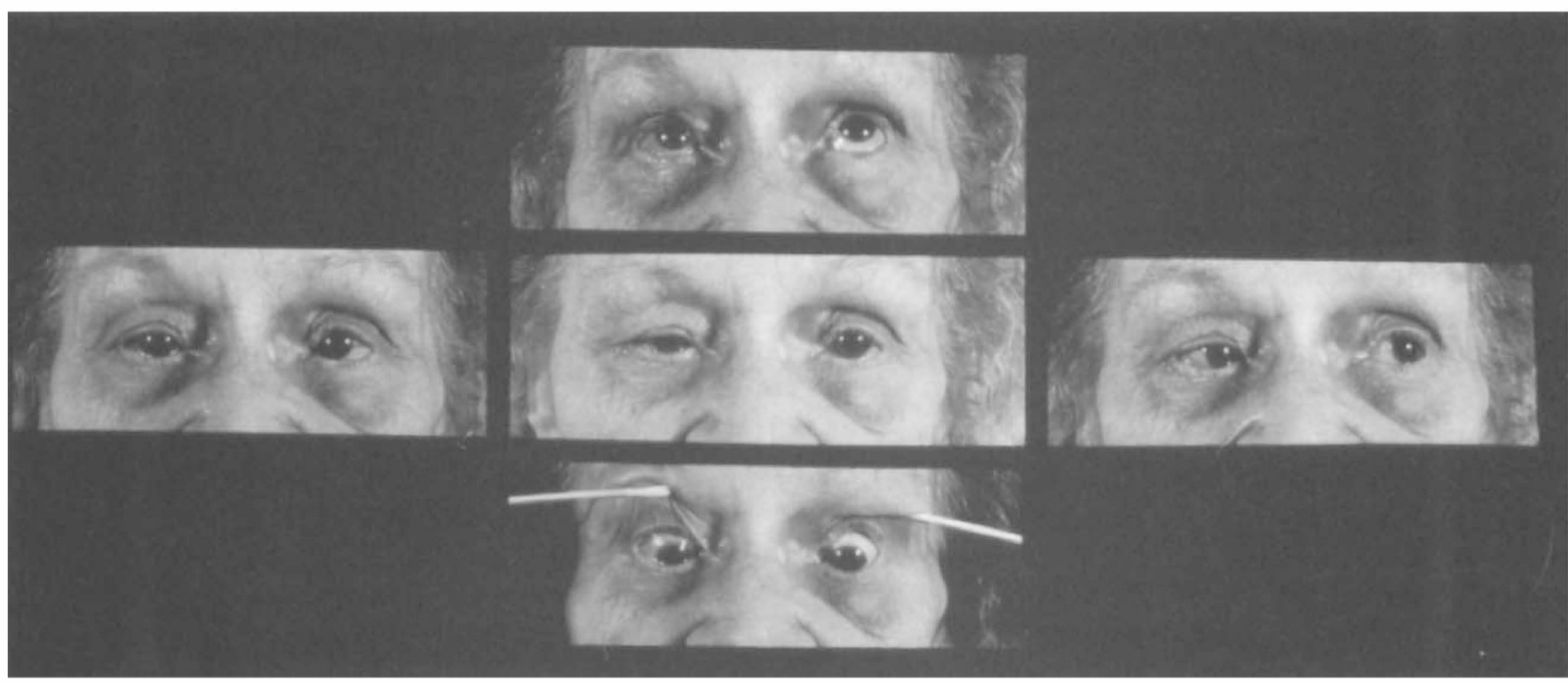

Fig. 1. Two days following right phacoemulsification, showing ptosis, exotropia and left hypertropia. Versions elicited absent abduction and limitation of elevation in the right eye. 


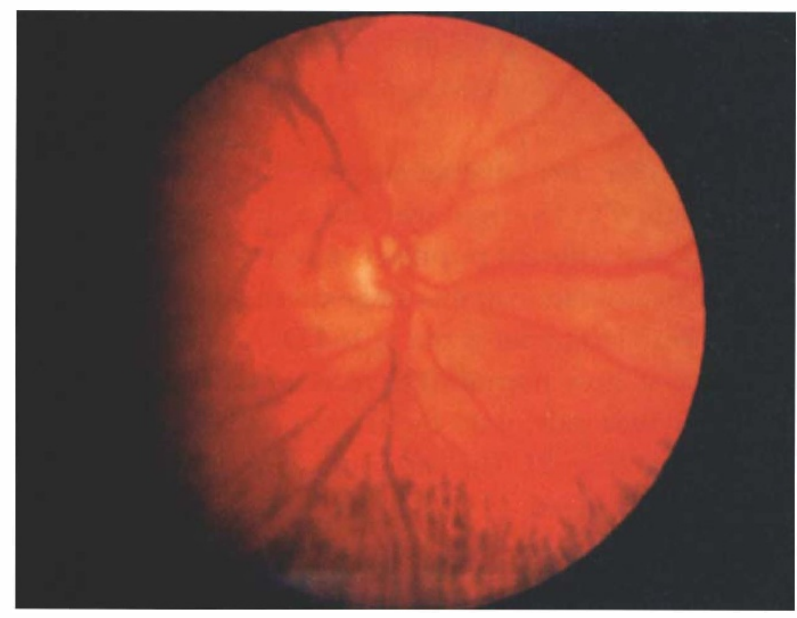

(a)

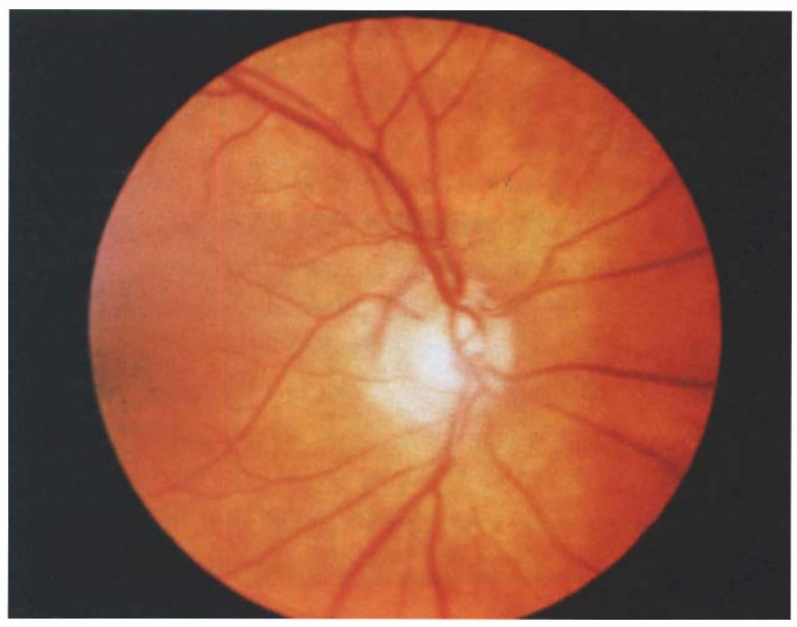

(b)

Fig. 2. Fundal view of the right eye after cataract surgery: (a) at 2 days showing a normal disc and (b) at 3 months demonstrating pallor of the inferior half of the disc.

the needle, or the optic nerve may have been damaged by adrenaline-induced vasospasm. Local anaesthetic agents have also been implicated in a myotoxic effect, ${ }^{3}$ causing a spectrum of manifestations ranging from paresis to contracture of the antagonist or the affected muscle. The ptosis and left hypertropia are explained by paresis of the levator and superior rectus muscles followed by secondary contracture of the antagonist inferior rectus. ${ }^{1,4}$ The other sequelae described by Capo and Guyton ${ }^{5}$ are transient paresis of the affected muscle followed by its overaction caused by segmental contracture acting as surgical resection. In our case this may explain the initial esotropia subsequently developing into exotropia due to lateral rectus involvement.

In summary, a complex clinical picture may rarely complicate retrobulbar anaesthesia. Topical, subconjunctival or sub-Tenon's anaesthesia would obviate these complications.

\section{References}

1. Hameed LM. Strabismus presenting after cataract surgery. Ophthalmology 1991;98:247-52.

2 Feibel RM. Current concepts in retrobulbar anesthesia. Surv Ophthalmol 1985;30:102-10.

3. Carlson BM, Emerich S, Komorowski TE, Rainin EA, Shepad BM. Extraocular muscle regeneration in primates.

Ophthalmology 1992;99:582-9.

4. Esswein MB, von Noorden GK. Paresis of a vertical rectus muscle after cataract extraction. Am J Ophthalmol 1993;116:424-30.

5. Capo H, Guyton DL. Ipsilateral hypertropia following cataract surgery. Ophthalmology 1996;103:721-30.

Jyoti Raina

Gregory S. Kosmorsky

Kenneth W. Wright

Department of Ophthalmology, A-31

Cleveland Clinic Foundation

9500 Euclid Avenue

Cleveland

Ohio, USA
Jyoti Raina, FRCS, FRCOphth

1, The Asters

Goffs Oak

Waltham Cross

Herts EN7 6SD, UK

Sir,

An unusual cause of an acute red eye

Numerous reports exist of lost hard contact lenses presenting as lumps in the upper lid and fornix. Recently a report of a soft contact lens presenting as an intraocular foreign body, following a perforating eye injury, was documented. ${ }^{1}$ To our knowledge there are no previous reports of lost soft contact lenses presenting as an acute red eye many years after discontinuation of wear.

\section{Case report}

A 43-year-old woman presented to the eye casualty with a 2-day history of discomfort in the left eye. On the third day she awoke with pain, redness and epiphora. Past ophthalmic enquiry elicited that she had worn soft contact lenses. Lens wear was discontinued 6 years previously because a tremor associated with multiple sclerosis caused difficulty handling the lenses.

Slit-lamp examination revealed a soft contact lens positioned over the temporal bulbar conjunctiva (Fig. 1). There was marked circumlimbal injection and a large corneal erosion. The anterior chamber was quiet and there was no corneal stromal infiltration. Corneal hypoaesthesia was not demonstrated on clinical testing.

Bacteriological investigations of the soft contact lens and conjunctival swab, including Acanthamoeba culture, were negative. Histopathological examination showed eosinophilic acellular material surrounding the lens (Fig. 2). 